\title{
Seedling Morphology of Some Medicinal Plants from Leguminosae of Tripura, North-East India
}

\section{Rashmi Rani*, Badal Kumar Datta}

Plant Taxonomy and Biodiversity Laboratory, Department of Botany, Tripura University, Suryamaninagar, 799022, Tripura.

Study Area: Suryamaninagar, India

Coordinates: $23.75^{\prime} \mathrm{N}$; $91.27^{\prime} \mathrm{E}$

Key words: Conservation Policy, Phenogram, Plant Lifecycle

\section{Introduction:}

Seedling is basically juvenile stages of a plant germinating from seed, as per Matheus \& Lopes (2007) seed and seedling morphological characterization is important for species differentiation and recognition at a young stage in the field. Seedling characters are important and reliable in the delimitation of species, genera and families. A critical examination of the correlation between a group of characters of both the adult as well as the juvenile plant has repeatedly shown results in better understanding of taxonomically difficult taxa. (Bokdam, 1977). The study of seedling morphology, which had been a less explored field in flowering plants, particularly in angiosperms, has now emerged as an essential discipline for taxonomic research at present. So, proper studies on seedling and their morphology will be helpful for their early identification and conservation of natural resources which in turn shall help in the conservation of biodiversity. Seedling characteristics of Pongamia pinnata (L.) Pierre has been studied by Khan et al. (2015). Seedling morphology in the identification of some Indian species of Bauhinia L. (Caesalpiniaceae) has been studied by Das and Paria (1999). Roy \& Datta (2014) studied Early identification and phnetic analysis of eight species of subtribe Cassiineae (Leguminosae: Caesalpinioideae). Seedling morphology is a less explored work in India, so in this study, an attempt has been made to study seedling morphology of some Leguminosae plants. In this study Abrus precatorius L., Bauhinia acuminata L., Caesalpinia bonduc (Linnaeus) Roxburgh, Caesalpinia pulcherrima (L.) Sw., Cajanus scarabaeoides (L.) Thouars, Calopogonium mucunoides Desv., Canavalia gladiata (Jacq.) DC., Clitoria ternatea L.,

\section{Abstract}

Seedling characters are important and promising from the taxonomic as well as evolutionary point of view. Seedling morphology of twenty-two medicinal plants of Leguminosae was studied, collected from different parts of Tripura, North-east India. The investigation was based on the germination pattern, position of cotyledon, hypocotyl, epicotyl, and paracotyledon and eophylls development. An artificial key has been constructed for easier identification of plants in their juvenile stage. Phenogram has been prepared to investigate the correlation between the investigated taxa.

Crotalaria spectabilis Roth, Crotalaria pallida Aiton, Crotalaria verrucosa L., Desmodium gangeticum (L.) DC., Desmodium heterocarpon (L.) DC., Desmodium triflorum (L.) DC., Mimosa pudica L., Mucuna bracteata DC., Phyllodium pulchellum (L.) Desv., Pueraria montana var. chinensis (Ohwi) Sanjappa \& Pradeep, Pueraria phaseoloides (Roxb.) Benth., Tephrosia purpurea (L.) Pers., Uraria crinita (L.) DC., and Uraria rufescens (DC.) Schindl have been investigated based on seedling characteristics. The key prepared in the present study is completely a new initiative for the early identification and conservation of the studied taxa. The phenogram presented in this study is the first report of the implication of seedling morphological character in the phenetic analysis of the members of the family Leguminosae found in Tripura.

\section{Methodology:}

Tripura is a state in North-East India and considered as a biodiversity hotspot. The state lies between $22^{\circ} 56^{\prime}$ to $24^{\circ}$ $32^{\prime}$ North latitudes and $91^{\circ} 09^{\prime}$ to $92^{\circ} 20^{\prime}$ East longitudes with an aerial extent of 10,491.69 sq. $\mathrm{km}$.

The seedling specimens Abrus precatorius L., Bauhinia acuminata L., Caesalpinia bonduc (Linnaeus) Roxburgh, Caesalpinia pulcherrima (L.) Sw., Cajanus scarabaeoides (L.) Thouars, Calopogonium mucunoides Desv., Canavalia gladiata (Jacq.) DC., Clitoria ternatea L., Crotalaria spectabilis Roth, Crotalaria pallida Aiton, Crotalaria verrucosa L., Desmodium gangeticum (L.) DC., Desmodium heterocarpon (L.) DC., Desmodium triflorum (L.) DC., Mimosa pudica L., Mucuna bracteata DC., Phyllodium pulchellum (L.) Desv., Puerariamontana var. chinensis (Ohwi) Sanjappa \& Pradeep, Pueraria phaseoloides (Roxb.) Benth., Tephrosia purpurea (L.) Pers., 
Uraria crinita (L.) DC., and Uraria rufescens (DC.) SchindlL were collected from different localities of Tripura. The specimens were photographed and documented in the form of herbarium sheets. They were compared and identified with the help of seedling raised from identified seeds. At least eight to ten specimens of each growth form were studied from various habitats. Morphological observation and description of seedlings were done according to Duke (1965), Burger (1972), Bokdam (1977), Vogel (1980) and Paria (2014). Artificial keys were prepared for the identification of investigated taxa in the juvenile stage. Phenogram was prepared based on the UPGMA method in PAST software.

\section{Results and Discussion:}

Diagnoses and key to identification of the investigated taxa.

1. Abrus precatorius Linnaeus, Syst. Nat., ed. 12. 2: 472. 1767 .

Seedling Morphology: (Up to oth leaf stage)

Seedling PER. Roots tap, elongating. Hypocotyl epigeous, glabrous, elongating, soft. Paracotyledon epigeous, equal, sessile, exstipulate, obovate, apex obtuse to rounded, margin entire, base attenuate, venation pattern palmate, lamina balance symmetric. Epicotyl green, soft, terete, glabrous. Eophylls compound, opposite, leaflet shape oblong, leaflets 6 pairs, opposite, shape of lamina oblong, leaflet base obtuse, apex retuse, margin entire, venation pattern pinnate, the surface of blade glabrous both surface. Next leaves pinnately compound, paripinnate, oblong, alternate, oblong, obtuse, retuse, symmetric, entire, pinnate, other characters more or less the same as that of first two leaves except for measurements.

\section{Bauhinia acuminata Linnaeus, Sp. Pl. 1:376. 1753.} Seedling Morphology:(Up to 8th leaf stage)

Seedling PER. Roots tap, reduced. Hypocotyl epigeous, glabrous, elongating, soft. Paracotyledon epigeous, equal, sessile, stipulate, leaf elliptic, apex rounded, the margin of lamina entire, base cuneate, venation pattern palmate, the surface of blade glabrous both surface, lamina balance symmetric. Epicotyl green, soft, terete, hairy. Eophylls simple, alternate, leaf elliptic/lobed, base cordate, apex lobed, margin entire, venation pattern palmate, the surface of blade glabrous above, hairy below. Next leaves simple, elliptic/bilobed, alternate, cordate, lobed, symmetric, entire, palmate, other characters more or less the same as that of first two leaves except for measurements.

\section{Caesalpinia bonduc (Linnaeus) Roxburgh, Fl.} Ind., ed. 1832. 2:362. 1832.

Seedling Morphology:(Up to oth leaf stage)

Seedling CHR. Roots tap, elongating,. Cotyledon hypogeal, exstipulate, sessile, globose, apices rounded, base cuneate, margin entire. Epicotyl terete, glabrous. Eophylls pinnately compound, opposite, stipulate, petiolate, shape ovate, apex obtuse, base cordate, margin entire, venation pattern pinnate, Next leaves bipinnately compound, alternate, petiolate, terete, other characters more or less same as that of first two leaves except for measurements.

\section{Caesalpinia pulcherrima (Linnaeus) Swartz,}

Observ. Bot. 166. 1791.

Seedling Morphology:(Up to oth leaf stage)

Seedling PER. Roots tap, elongating. Hypocotyl epigeous, glabrous, elongating, soft. Paracotyledon epigeous, equal, petiolate, exstipulate, oblong, apex rounded to truncate, margin entire, base auriculate, venation pattern palmate, glabrous, both surface glabrous, lamina balance symmetric. Epicotyl green, soft, angular, glabrous. Eophylls pinnately compound; the paripinnate, opposite, stipulate, petiolate, shape of lamina oblong, base oblique, apex obtuse, margin entire, venation pattern pinnate. Next leaves bipinnately compound, stipulate, petiolate, alternate, oblong, oblique, obtuse, entire, pinnate, glabrous both surface, other characters more or less same as that of first two leaves except measurements.

\section{Cajanus scarabaeoides (Linnaeus) Thouars, Dict. Sci. Nat. 6:617.1817.}

Seedling Morphology: (Up to 8th leaf stage)

Seedling CHR. Roots tap, reduced. Hypocotyl hypogeous, glabrous, reduced, leathery. Cotyledon hypogeous, equal, inserted at a cotyledonary node, exstipulate, ovate, base truncate, apices rounded, margin entire. Epicotyl green, soft, terete, hairy. Eophylls simple, opposite, exstipulate, petiolate, leaf ovate, base cordate, apex acute, margin entire, venation patterns innate, the surface of blade hairy. Next leaves ternately compound, exstipulate, petiolate, alternate, leaflet elliptic, apex obtuse, base cuneate, entire, pinnate. Subsequent leaves ternately compound, elliptic, alternate, leaflet elliptic, other characters more or less the same as that of first two leaves except for measurements.

\section{Calopogonium mucunoides Desvaux, Ann. Sci. Nat. (Paris). 9:423. 1826.}

Seedling Morphology:(Up to 8th leaf stage)

Seedling PEF. Roots tap, short elongating. Hypocotyl epigeous terete, hairy, elongating, soft. Paracotyledon epigeous, opposite, sessile, exstipulate, leaf oblong, apex truncate, base cuneate, margin entire, venation pinnate, glabrous. Epicotyl light green, soft, terete, hairy. Eophylls simple, opposite, petiolate, leaf elliptic, apex truncate/retuse, base cordate, margin ciliate, venation pinnate, glabrous. Next leaves ternately compound, alternate, elliptic, apex obtuse, base cuneate, margin entire, venation pinnate. Subsequent leaves alternate, ternately compound, elliptic, other characters more or less the same as that of first two leaves except for measurements.

\section{Canavalia gladiata (Jacquin) Candolle, Prodr. 2: 404. 1825.}

Seedling Morphology:(Up to oth leaf stage)

Seedling PER. Roots tap, reduced. Hypocotyl epigeous, elongating, woody.Paracotyledon epigeous, equal, sessile, exstipulate, oblong, apex retuse, base cuneate,, margin entire, venation pattern pinnate. Epicotylgreen, woody, glabrous. Eophylls simple, opposite, stipulate, petiolate, leaf elliptic, apex mucronate, base auriculate, margin sinulate, venation pattern palmate. Next leaves ternately compound, stipulate, petiolate, alternate, ovate, apiculate, cuneate, entire, pinnate. Subsequent leaves ternately compound, ovate, stipulate, other characters more or less the same as that of first two leaves except for measurements.

8. Clitoria ternatea Linnaeus, Sp. Pl. 2: 753. 1753. Seedling Morphology: (Up to oth leaf stage) 
Seedling PER. Roots tap, short elongating. Hypocotyl epigeous, hairy, elongating, soft.Paracotyledon epigeous, equal, petiolate, leaf elliptic, $\pm 2-2.5 \times \pm 1.4-1.6 \mathrm{~cm}$, apex rounded, base cordate, margin entire, venation pattern palmate. Epicotyl green, soft, hairy. Eophylls simple, opposite, stipulate, petiolate leaf elliptic, apex mucronate, base cuneate, margin entire, venation patterns innate.Next leaves ternately compound, stipulate, petiolate, alternate, elliptic, other characters more or less the same as that of first two leaves except for measurements.

\section{Crotalaria pallida Aiton, Hort. Kew.3:20.1789.}

Seedling morphology:(Up to 1oth leaf stage).

Seedling PEF. Roots Tap, elongating. Hypocotyl epigeous, hairy, elongating. Paracotyledon epigeous, equal, petiolate, exstipulate, leaf shape oblong, apex rounded, base obtuse, margin entire, venation pattern pinnate. Epicotyl green, moderately woody. Eophylls ternately compound, alternate, stipulate, petiolate, leaf shape obovate, apex obtuse, base cuneate, margin entire, venation pattern pinnate. Next leaves ternately compound,/trifoliate, alternate, other characters more or less the same as that of first two leaves except for measurements.

10. Crotalaria spectabilis Roth, Nov. Pl. Sp.341. 1821. Seedling morphology: (Up to oth leaf stage)

Seedling PEF. Roots tap, elongating. Hypocotyl epigeous, terete, elongating. Paracotyledon epigeous, equal, symmetric, petiolate, exstipulate, leaf shape oblong, apex obtuse, base cordate, margin entire, venation pattern palmate, Epicotyl green, soft. Eophylls simple, alternate, petiolate, leaf shape obovate, apex, obtuse, base cuneate, margin entire, venation pattern pinnate. Next leaves simple, alternate, soft, petiolate, other characters more or less the same as that of first two leaves except for measurements.

11. Crotalaria verrucosa Linnaeus, Sp. Pl. 2: 715 . 1753. Seedling Morphology: (Up to oth leaf stage)

Seedling PEF. Roots tap, short elongating. Hypocotyl elongating, soft. Paracotyledon epigeous, equal, petiolate leaf elliptic, apex round, base cuneate, margin entire, venation pattern palmate. Epicotyl green, terete. Eophylls simple, alternate, exstipulate, petiolate, leaf elliptic, apex acuminate, base cuneate, margin entire/ciliate, venation pattern pinnate. Next leaves simple, elliptic, exstipulate, other characters more or less the same as that of first two leaves except for measurements.

\section{Desmodium gangeticum (Linnaeus) Candolle, Prodr. 2:327. 1825.}

Seedling Morphology: (Up to 12th leaf stage)

Seedling PEF. Roots tap, reduced. Hypocotyl epigeous, reduced. Paracotyledon epigeous, petiolate, exstipulate, leaf elliptic, apex obtuse, base cuneate, margin entire, venation pinnate. Epicotyl green, moderately woody. Eophylls simple, opposite, stipulate, petiolate, leaf elliptic, apex rounded/truncate, base cordate, the margin of lamina entire, venation pattern pinnate. Third and fourth leaves simple, alternate, stipulate, petiolate, leaf ovate/elliptic, base cordate/rounded, apex emarginate. Next leaves leaf oblong, apex acute, base cordate, venation same other characters more or less the same as that of first two leaves except for measurements.

\section{Desmodium heterocarpon (Linnaeus) Candolle, Prodr. 2:337. 1825. \\ Seedling Morphology:(Upto 12th leaf stage) \\ Seedling PEF. Roots tap, elongating. Hypocotyl epigeous,}

reduced, woody. Paracotyledon epigeous, equal, sessile, exstipulate leaf elliptic, apex rounded, base cuneate/oblique, margin entire, venation palmate. terete, hairy. Eophylls simple, opposite, stipulate, petiolate, leaf obovate, apex emarginate, base truncate/ cordate, margin entire, venation pattern pinnate. Next leaves simple, oblong, stipulate, petiolate, other characters more or less the same as that of first two leaves except for measurements.

\section{Desmodium triflorum (Linnaeus) Candolle, Prodr. 2:334. 1825.}

Seedling Morphology:(Upto 12th leaf stage)

Seedling PEF. Roots tap, reduced. Hypocotyl epigeous, reduced, soft. Paracotyledon epigeous, equal, petiolate, exstipulate, leaf oblong, apex obtuse, base cuneate, margin entire, venation pattern pinnate. Epicotylgreen, soft, terete. Eophylls simple, opposite, exstipulate, petiolate, leaf elliptic, apex emerginate, base cordate, margin entire, venation pattern pinnate. Third and fourth leaves simple, elliptic, soft, exstipulate, alternate, cordate, emarginate, entire, pinnate. Eight and subsequent leaves ternately compound, exstipulate, petiolate, other characters more or less the same as that of first two leaves except for measurements.

15. Mucuna pruriens (L.) DC.

Seedling morphology:(Up to oth leaf stage)

Seedling CHR. Roots tap, short elongating. Hypocotyl hypogeous, reduced. Cotyledon cryptocotylar, exstipulate, cotyledon shape ovate, apex obtuse, base cuneate, margin entire. Epicotyl terete, hairy. Eophylls simple, opposite, stipulate, petiolate, leaf shape ovate, apex acuminate, base auriculate, margin sinulate, venation pattern pinnate. Next leaves ternately compound, stipulate, petiolate, alternate, ovate, cuneate, apiculate, entire. Subsequent leaves ternately compound, ovate, alternate, other characters more or less the same as that of first two leaves except for measurements.

\section{Mimosa pudica Linnaeus, Sp. Pl. 1:518. 1753.}

Seedling Morphology:(Up to oth leaf stage)

Seedling PEF. Roots tap, elongating, unbranched. Hypocotyl epigeous, elongating, soft. Paracotyledon epigeous, equal, petiolate, leaf oblong, $\pm 0.4-0.6 \times \pm 0.4-0.6$, apex retuse, base cordate, margin entire, venation pattern pinnate. Epicotylterete, glabrous. Eophylls pinnately compound, alternate, leaf oblong, stipulate, petiolate, leaf obovate, apex obtuse, base cuneate, margin entire, venation pattern palmate. Next leaves pinnately, compound, alternate, stipulate, petiolate, obovate, cuneate, obtuse, other characters more or less the same as that of first two leaves except for measurements.

\section{Phyllodium pulchellum (Linnaeus) Desvaux, J. Bot. Agric. 1: 124. 1813.} Seedling Morphology:(Up to oth leaf stage)

Seedling PEF. Roots tap, short elongating. Hypocotyl epigeous, reduced, soft. Paracotyledon epigeous, equal, petiolate, leaf oblong, apex rounded, base cuneate, margin entire, venation pattern pinnate. Epicotyl terete, hair. Eophylls simple, opposite, exstipulate, petiolate, leaf elliptic, apex retuse, base cordate, margin entire, venation pattern pinnate. Next leaves ternately compound, exstipulate, petiolate, alternate, elliptic, apex obtuse, base obtuse/cordate, margin entire, venation pattern pinnate. Subsequent leaves ternately compound, alternate, elliptic, other characters more or less the same as that of first two leaves except for measurements. 
18. Puerariamontana var. chinensis (Loureiro) Merrill, Trans. Amer. Philos. Soc., ser. 2. 24(2): 10, 210.1935.

Seedling morphology:(Up to 1oth leaf stage)

Seedling CHR.Roots tap, elongating. Hypocotyl hypogeous, reduced, soft. Cotyledon two, cryptocotylar, petiolate, cotyledon shape ellipsoidal, apex rounded, base cordate, margin entire. Epicotyl terete, hairy. Eophylls ternately compound, alternate, stipulate, petiolate, leaf shape ovate, apex acute, base obtuse, margin entire, venation pattern palmate. Next leaves ternately compound, alternate, ovate, other characters more or less the same as that of first two leaves except for measurements.

\section{Pueraria phaseoloides (Roxb.) Benth. J. Linn. Soc.,} Bot. 9: 125. 1865.

Seedling morphology: (Up to 1oth leaf stage)

Seedling PEF. Roots tap, short elongating. Hypocotyl epigeous,reduced, soft. Paracotyledon epigeous, equal, sessile, exstipulate, leaf shape oblong, apex obtuse, base cuneate, margin entire, venation pattern pinnate. Epicotyl terete, hairy. Eophylls simple, opposite, exstipulate, petiolate, leaf shape elliptic, apex acute, base obtuse, margin ciliate, venation pattern pinnate. Next leaves ternately compound, alternate, stipulate, petiolate, elliptic. Subsequent leaves ternately compound, alternate, elliptic, other characters more or less the same as that of first two leaves except for measurements.

2o. Tephrosia purpurea (Linnaeus) Persoon, Syn. Pl. 2:329. 1807 .

Seedling Morphology:(Up to 1oth leaf stage)

Seedling PEF. Roots tap, short elongating. Hypocotyl epigeous, elongating, soft.Paracotyledon epigeous, equal, petiolate, exstipulate, leaf elliptic, apex obtuse, base obtuse, margin entire, venation pattern pinnate.Epicotyl terete, hairy. First leaf simple, alternate, stipulate, petiolate, leaf elliptic, apex obtuse,base cuneate, margin entire, venation pattern pinnate. Second leaf ternately compound, alternate. Next leaves ternately compound, alternate, leaflet obovate, other characters more or less the same as that of first two leaves except for measurements.

\section{Uraria crinita (Linnaeus) Desvaux ex Candolle, Prodr. 2:324. 1825.}

Seedling Morphology:(Up to oth leaf stage)

Seedling PEF. Roots tap, elongating. Hypocotyl epigeous, reduced, soft. Paracotyledon epigeous, equal, petiolate, exstipulate, leaf oblong, apex obtuse, base cuneate, margin entire, venation pattern pinnate. Epicotyl terete, hairy. Eophylls simple, opposite, stipulate, petiolate, leaf elliptic, apex emarginate, base truncate/cordate, margin entire, venation pattern pinnate. Next leaves simple, elliptic, alternate, other characters more or less the same as that of first two leaves except for measurements.

\section{Uraria rufescens (Candolle) Schindler, Repert. Spec. Nov. Regni Veg. 21:14. 1925.}

Seedling Morphology: (Up to 1oth leaf stage)

Seedling PEF. Roots tap, reduced. Hypocotyl epigeous, short elongating, soft. Paracotyledon epigeous, equal, symmetric, petiolate, exstipulate, leaf oblong, apex obtuse, base cuneate, margin entire, venation pattern pinnate.Epicotylterete, hairy. Eophylls simple, opposite, stipulate, petiolate, leaf oblong/elliptic, apex truncate, base truncate, margin entire, venation pattern pinnate. Next leaves simple, obovate, other characters are more or less the same as that of first two leaves except for measurements.

\section{Key to the investigated taxa}

1 a. (Para)cotyledons exstipulate.....................................................

b. (Para)cotyledons stipulate........................................................21

2 a. (1) (Para)cotyledons always phanerocotylar............................. 3

b. (Para) cotyledons always cryptocotylar...................................18

3 a. (2) Paracotyledons are always foliaceous type........................4

b. Paracotyledons are always reserve type.................................15

4 a. (3) Leaves either simple or compound, both never present together.

b. Both simple and compound leaves present............................11

5 a. (4)Leaves always compound, never simple... Crotalaria pallida

b. Leaves always simple never compound...................................6

6 a. (5)Eophylls and subsequent leaves have always alternate phyllotaxy....................................................................................

b. Eophylls have opposite phyllotaxy and subsequent leaves have alternate phyllotaxy............................................................8

7 a. (6)Leaves are elliptic............................. Crotalaria verrucosa

b. Leaves are obovate................................ Crotalaria spectabilis

8 a. (6) Paracotyledon leaf shape is elliptic.................................. 9

b. Paracotyledon leaf shape is oblong.........................................10

9 a. Eophylls are elliptic.

b. Eophylls are obovate......................Desmodium heterocarpon

10a. (8) Leaves are elliptic........................................... Uraria crinita

b. Leaves are obovate......................................... Uraria rufescens

11 a. Eophylls and subsequent leaves have always alternate phyllotaxy Tephrosia purpurea

b. Eophylls have opposite phyllotaxy and subsequent leaves have alternate phyllotaxy

12a. (11)Hypocotyl elongating........... Calopogonium mucunoides

b. Hypocotyl reduced...

13a. (12)Epicotyl glabrous.

b. Epicotyl hairy..... Desmodium triflorum

4a. (13)Hypocotyl hairy................................. Phyllodium pulchellum

b. Hypocotyl glabrous Pueraria phaseoloides

15a. (3)Eophylls always simple and subsequent leaves are compound

16

b. Eophylls and subsequent leaves are always compound......17

16a. (15) Paracotyledons are elliptic. Clitoria ternatea

b. (15)Paracotyledons are oblong................. Canavalia gladiata

17a. Paracotyledon leaf shape are oblong... Caesalpinia pulcherrima

b. Paracotyledon leaf shape are obovate....... Abrus precatorius

18a. (2)Eophylls and subsequent leaves are ternately compound.

b. Eophylls are simple and subsequent leaves are compound in nature. 20

19a. (18) Eophylls are alternate. .Pueraria montana

b. Eophylls are opposite. 2oa. (18)Leaves are elliptic

b. Leaves are ovate. Caesalpinia bonduc

21a. (1)Leaves are simple. Cajanus scarabaeoides

b. Leaves are compound.

In this investigation, twenty-two species belonging to fourteen genera of family Leguminosae has been described based on seedling morphological characters. These 


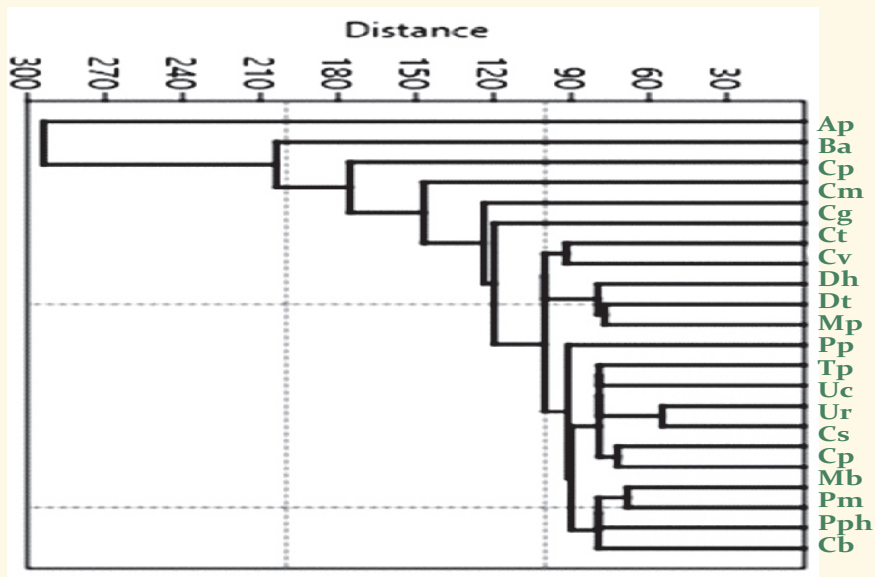

Figure: 2- Phenogram of the investigated members of the family Leguminosae using UPGMA analysis. Abbreviations: Ap- Abrus precatorius, Ba-Bauhinia acuminata,Cb-Caesalpinia bonduc, CpCaesalpinia pulcherrima, Cs-Cajanus scarabaeoides, CmCalopogonium mucunoides, Cg-Canavalia gladiata, Ct- Clitoria ternatea, $\mathrm{Cp}$-Crotalaria pallida, Cs-Crotalaria spectabilis, $\mathrm{Cv}$-Crotalaria verrucosa, Dg-Desmodium gangeticum, Dh-D. hetero carpon, Dt- D. triflorum, Mp-Mimosa pudica, Mb-Mucuna bracteata, Pp-Phyllodium pulchellum, Pm-Pueraria montana, Pph-Pueraria phaseoloides, TpTephrosia purpurea, Uc-Uraria crinita, Ur-U. rufescens.

seedling characters can serve as a marker character for identification of taxa. Out of twenty-two studied taxa four species (Cajanus scarabaeoides, Mucuna bracteata, Pueraria montana, Caesalpinia bonduc) showed Cryptocotylar Hypogeal Reserve (CHR) type of germination and rest 18 species showed Phanerocotylar Epigeal Foliaceous (PEF) type of germination. PEF type of germination is advantageous over other types of germination as they generally grow faster than when exposed to increased light (Pooma \& Bongers, 1988). Multivariant phenetic analyses have been used in classifying many plants and interpreting results of taxonomic studies.(Sneath \& Sokal, 1973). Sanyal \& Paria (2014) studied the seedling morphology of twenty-five species of Leguminosae. Seedling characters like germination pattern, nature of root, hypocotyl, shape, size, colour, apex, base, the margin of paracotyledons and eophylls has been considered in this investigation. The root of Bauhinia acuminata, Cajanus scarabaeoides, Canavalia gladiata, Desmodium gangeticum, Desmodium triflorum and Uraria rufescens is reduced, Calopogonium mucunoides, Clitoria ternatea, Crotalaria verrucosa, Phyllodium pulchellum, Pueraria phaseoloides, Tephrosia purpureais short elongating while Abrus precatorius, Caesalpinia pulcherrima, Crotalaria pallida, Crotalaria spectabilis, Caesalpini abonduc, Desmodium heterocarpon Mimosa pudica, Mucuna bracteata, Pueraria montana and Uraria crinita have elongating roots. Hypocotyl of Abrus precatorius, Bauhinia acuminata, Caesalpinia pulcherrima, Calopogonium mucunoides, Canavalia gladiata, Clitoria ternatea, Crotalaria pallida, Crotalaria spectabilis, Crotalaria verrucosa, Mimosa pudica, and Tephrosia
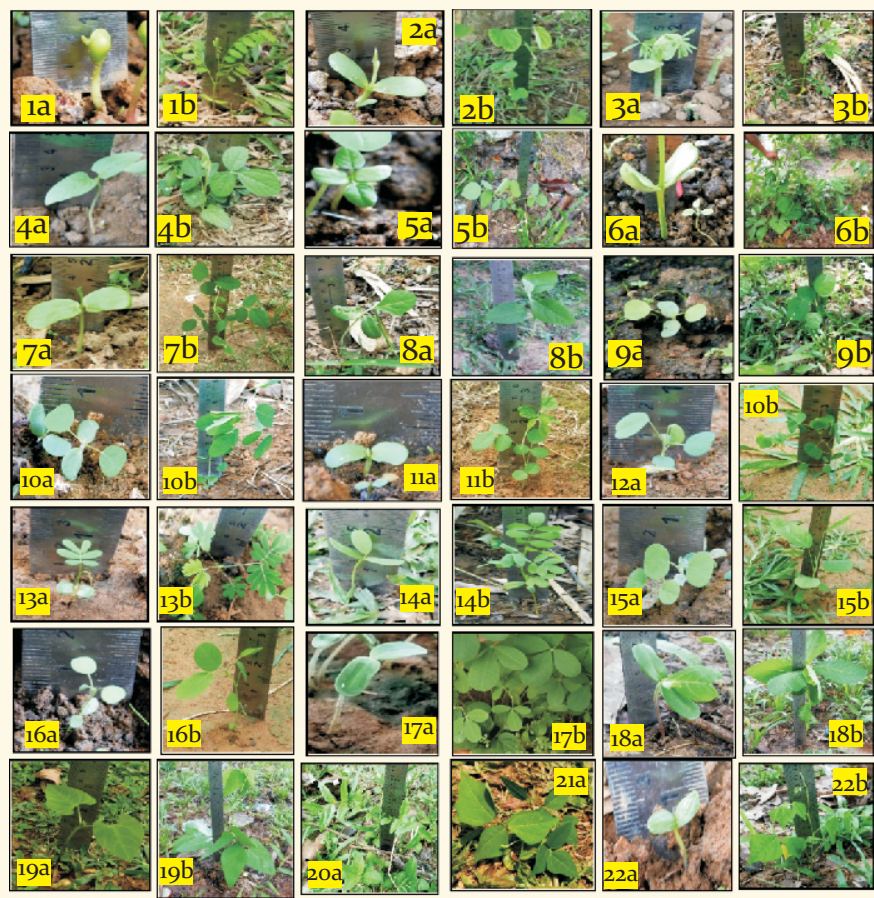

Plate-1: (1)Abrus precatorius (2)Bauhinia acuminata (3)Caesalpinia pulcherrima (4)Cajanus scarabaeoides (5)Calopogonium mucunoides (6)Canavalia gladiata (7)Clitoria ternatea (8)Crotalaria verrucosa (9)Desmodium gangeticum (10)Desmodium heterocarpon (11)Phyllodium pulchellum (12)Desmodium triflorum (13)Mimosa pudica (14)Tephrosia purpurea (15)Uraria crinita (16)Uraria crinita (17)Crotalaria pallida (18)Crotalaria spectabilis (19)Mucuna bracteata (20)a Caesalpinia bonduc (21)Pueraria montana (21) Pueraria phaseoloides (a=Paracotyledon; $\mathbf{b}=$ Mature seedling)

purpurea was elongating, Uraria rufescens had short elongating while Cajanus scarabaeoides, Desmodium gangeticum, Caesalpiniabonduc, Desmodium heterocarpon, Desmodium triflorum, Mucuna bracteata, Phyllodium pulchellum, Puerariamontana, Pueraria phaseoloides, and Uraria crinita has reduced hypocotyl. Bauhinia acuminata, Clitoria ternatea, Crotalaria verrucosa, Desmodium gangeticum, Desmodium heterocarpon, Puerariamontana, Tephrosia purpurea has elliptic paracotyledon, Caesalpinia pulcherrima, Calopogonium mucunoides, Canavalia gladiata, Crotalaria pallida, Crotalaria spectabilis, Desmodium triflorum, Desmodium triflorum, Phyllodium pulchellum, Pueraria phaseoloides, Uraria crinitaand Uraria rufescenshas oblong paracotyledon, Cajanus scarabaeoides, Mucuna bracteata had ovate paracotyledon. Eophylls have either opposite (Caesalpinia pulcherrima, Calopogonium mucunoides etc.) or alternate (Crotalaria pallida, Crotalaria spectabilis, Mimosa pudica etc) phyllotaxy. Based on these seedling characters artificial key has been constructed for the easier identification of plants in their juvenile stage. A phenogram has been constructed using UPGMA method to draw a correlation between the taxa. Phenogram shows all three Desmodium species are closely related and are present in one cluster. Pueraria montana, Pueraria phaseoloides are closely related based on seedling 
character and are present in one cluster. Similarly Uraria sp. and Crotalaria species are present in their respective cluster.

Conclusively, the seedling character of Leguminosae plant is related to each other and the artificial key is extremely useful for early identification and restoration of plants. Seedling morphological studies of medicinal plants help to restore the wild medicinal plants in the early stage of their life cycle. Therefore from the conservation point, identification of seedlings of medicinal plants can help to prevent or restore the plant source loss in the Tripura and can also help in developing strategies for protection, conservation and management of medicinal plant of Tripura.

Acknowledgement:

Author is grateful to Tripura University for providing RET fellowship

\section{References:}

Bokdam, J. (1977): Seedling morphology of some African Sapotaceae and its taxonomical significance. Pub. by: Mendelllingen Landouwhoge School, Wegeningen, Netherland.

Burger, D. (1972): Seedlings of some Tropical Trees and Shrubs Mainly of South East Asia. Pub. by: Centre for Agricultural Publishing and Documentation, Wageningen.

Das, D.C. \& Paria, N.D. (1999): Seedling morphology in identification of some Indian species of Bauhinia L. (Caesalpiniaceae). Feddes Repertorium. 110(5-6):375-379.
De Vogel, E.F. (1980): Seedlings of Dicotyledons: Structure, Development, types, description of 150 Woody Malesian Taxa. Pub. by: Centre for Agricultural Publishing and Documentation, Wageningen.

Duke, J.A. (1965): Keys for identification of seedling of some prominent woody species in eight forest types in Puerto Rico. Ann. Missouri Bot. Garden. 52:314-350.

Khan, D., Zaki, M.J., Shaukat, S.S. \& Sahito, Z.A. (2015): Seedling characteristics of Pongamia pinnata (1.) Pierre (Papilionaceae). Int.J. Biol., 12(3):457-479.

Matheus, M.T. \& Lopes, J.C. (2007): Fruit, seed and seedling morphology and seed germination in Erythrina variegata L. Rev. Bras. Sementes, 27(3):8-12.

Paria, N.D. \& Bose, A. Seedling morphology with reference to documentation and management of phytodiversity. In: Biodiversity and Livelihood: Proc. Nat. Conf. Biodiver. West Bengal Biodiversity Board, West Bengal. 31-36.

Pooma, J. \& Bongers, F. (1988): The effect of canopy gaps on growth and morphology of seedlings of rain forest species. Oecologia. 75: 625-632.

Roy, M. \& Datta, B.K. (2014): Early identification and honetic analysis of eight species of subtribe Cassiineae (Leguminosae: Caesalpinoideae) found in Tripura in relation to their seedling morphology. Pleione, 8(2):234-244.

Sanyal, S. \& Paria, N.D. (2014): Seedling morphology as a tool for taxonomic study in some members of Leguminosae (Fabaceae).Int.J. Plant Animal Environ. Sci., 5(1):1-15.

Sneath, P.H.A. \& Sokal, R.R. (1973): Numerical Taxonomy. Pub. by:W.H. Freeman and Co., San Fransisco. 